\title{
Electron spin evolution induced by interaction with nuclei in a quantum dot
}

\author{
Alexander Khaetskii ${ }^{1}$, Daniel Loss ${ }^{1}$, and Leonid Glazman ${ }^{2}$ \\ 1 Department of Physics and Astronomy, University of Basel, \\ Klingelbergstrasse 82, CH-4056 Basel, Switzerland and \\ 2 Theoretical Physics Institute, University of Minnesota, Minneapolis, MN 55455, USA.
}

\begin{abstract}
We study the decoherence of a single electron spin in an isolated quantum dot induced by hyperfine interaction with nuclei for times smaller than the nuclear spin relaxation time. The decay is caused by the spatial variation of the electron envelope wave function within the dot, leading to a nonuniform hyperfine coupling $A$. We show that the usual treatment of the problem based on the Markovian approximation is impossible because the correlation time for the nuclear magnetic field seen by the electron spin is itself determined by the flip-flop processes. The decay of the electron spin correlation function is not exponential but rather power (inverse logarithm) law-like. For polarized nuclei we find an exact solution and show that the precession amplitude and the decay behavior can be tuned by the magnetic field. The decay time is given by $\hbar N / A$, where $N$ is the number of nuclei inside the dot. The amplitude of precession, reached as a result of the decay, is finite. We show that there is a striking difference between the decoherence time for a single dot and the dephasing time for an ensemble of dots.
\end{abstract}

PACS numbers: PACS numbers: 85.35.Be; 73.21.La; 76.20.+q; 76.60.Es

\section{INTRODUCTION}

The spin dynamics of electrons in semiconducting nanostructures has become of central interest in recent years [1, 2]. The controlled manipulation of spin, and in particular of its phase, is the primary prerequisite needed for novel applications in conventional computer hardware as well as in quantum information processing. It is thus desirable to understand the mechanisms which limit the spin phase coherence of electrons, in particular in GaAs semiconductors, which have been shown [3] to exhibit unusually long spin decoherence times $T_{2}$ exceeding 100 ns. Since in GaAs each nucleus carries spin, the hyperfine interaction between electron and nuclear spins is unavoidable, and it is therefore important to underdffect on the electron spin dynamics [4]. This is particularly so for electrons which are confined to a closed system such as a quantum dot with a spin $1 / 2$ ground state, since, besides fundamental interest, these systems are promising candidates for scalable spin qubits [5]. For recent work on spin relaxation (characterized by $T_{1}$ times) in GaAs nanostructures we refer to Refs. [6, [7, 8].

Motivated by this we investigate in the following the spin dynamics of a single electron confined to a quantum dot in the presence of nuclear spins (see Fig. 1) [9]. We treat the case of unpolarized nuclei perturbatively, while for the fully polarized case we present an exact solution for the spin dynamics and show that the decay is non-exponential and can be strongly influenced by external magnetic fields. We use the term "decoherence" to describe the case with a single dot, and the term "dephasing" for an ensemble of dots [10]. The typical fluctuating nuclear magnetic field seen by the electron spin via the hyperfine interaction is of the order of 11 $\sim A /\left(\sqrt{N} g \mu_{B}\right)$, with an associated electron precession frequency $\omega_{N} \simeq A / \sqrt{N}$, where $A$ is a hyperfine constant, $g$ the electron g-factor, $\mu_{B}$ the Bohr magneton, and $N$ the number of nuclei inside the dot. For a typical dot size the electron wave function covers approximately $N=10^{5}$ nuclei, then this field is of the order of 100 Gauss in a GaAs quantum dot. The nuclei in turn interact with each other via dipolar interaction, which does not conserve the total nuclear spin and thus leads to a change of a given nuclear spin configuration within the time $T_{n 2} \approx 10^{-4} s$, which is just the period of precession of a nuclear spin in the local magnetic field generated by its neighbours.

We note that there are two different regimes of interest, depending on the parameter $\omega_{N} \tau_{c}$, where $\tau_{c}$ is the correlation time of the nuclear magnetic field $\mathbf{H}_{N}(t)$ seen by the electron spin via the hyperfine interaction. The simplest case is the perturbative regime $\omega_{N} \tau_{c} \ll 1$, characterized by dynamical narrowing: different random nuclear configurations change quickly in time, and, as a result, the spin dynamics is diffusive with a dephasing time $\simeq 1 /\left(\omega_{N}^{2} \tau_{c}\right)$. This case is realized, for example, for a system of quantum dots (or shallow donors) when the hopping rate $1 / \tau_{c}$ of the electron between neighbouring dots is high. The problem of electron spin relaxation for the case of electron hopping between shallow donors in GaAs was studied in Ref. 12. Using a perturbative approach, we easily obtain the following formulas for the longitudinal $\left(T_{1}\right)$ and transverse $\left(T_{2}\right)$ spin relaxation times [12, 13]: 


$$
\begin{array}{r}
\frac{1}{T_{1}}=\frac{\left(g \mu_{B}\right)^{2}}{\hbar^{2}} \frac{1}{2} \int_{-\infty}^{+\infty} d \tau \exp \left(-i \omega_{z} \tau\right) \operatorname{Re}\left[<H_{N x}(0) H_{N x}(\tau)>+<H_{N y}(0) H_{N y}(\tau)>\right] \\
\frac{1}{T_{2}}=\frac{1}{2 T_{1}}+\frac{\left(g \mu_{B}\right)^{2}}{\hbar^{2}} \frac{1}{2} \int_{-\infty}^{+\infty} d \tau<H_{N z}(0) H_{N z}(\tau)>
\end{array}
$$

where $\omega_{z}=g \mu_{B} B / \hbar$ is the Larmor spin precession frequency in the external magnetic field $B$ directed along the z-axis, and $\langle\ldots\rangle$ means the ensemble average of the fluctuating nuclear magnetic field correlators. (In the limit $\omega_{z} \tau_{c} \ll 1$ we obtain from the above formulas the well known result $T_{1}=T_{2}$, where we took into account the fact that the fluctuating nuclear field is isotropic.)

A more difficult situation arises when $\omega_{N} \tau_{c} \gg 1$, which requires a nonperturbative approach. It is this regime that we will consider in this paper, i.e. the electron is localized in a quantum dot, and the correlation time is due to the internal nuclear spin dynamics, i.e., $\tau_{c}=T_{n 2}$, giving $\omega_{N} \tau_{c}=10^{4}$. Thus, no usual treatment and no Markov approximation are possible. In particular, the perturbative formulas for $T_{1}, T_{2}$ (see Eqs.(1)) are not applicable here. Next, we need to address the important issue of averaging over different nuclear spin configurations in a single dot. Without internal nuclear spin dynamics, i.e. $T_{n 2} \rightarrow \infty$, no averaging is indicated. However, each flip-flop process (due to hyperfine interaction) creates a different nuclear spin configuration, and because of the spatial variation of the hyperfine coupling constants inside the dot, this leads to a different random value of the nuclear field seen by the electron spin and thus to its decoherence. Below we will find that this decoherence is non-exponential, but still we can indicate the characteristic time given by [10] $(A / \hbar N)^{-1}$. Moreover, we shall find that $T_{n 2} \gg(A \overline{\hbar N})^{-1}$, and thus still no averaging over the nuclear configurations is indicated (and dipolar interactions will be neglected henceforth). To underline the importance of this point, we will contrast below the unaveraged correlator with its average.

\section{UNPOLARIZED NUCLEI.}

We consider a single electron in an orbital ground state of a quantum dot. Its spin $\mathbf{S}$ couples to an external mag- netic field $\mathbf{B}$ and to nuclear spins $\left\{\mathbf{I}^{i}\right\}$ via hyperfine contact interaction, described by the Hamiltonian

$$
\hat{\mathcal{H}}=g \mu_{B} \mathbf{S} \cdot \mathbf{B}+\mathbf{S} \cdot \mathbf{h}_{N}, \mathbf{h}_{N}=\sum_{i} A_{i} \mathbf{I}^{i}=g \mu_{B} \mathbf{H}_{N},
$$

where $\mathbf{H}_{N}$ is the nuclear field. Note that the sum in Eq.(2) runs over the entire space. The coupling constant with the i-th nucleus, $A_{i}=A v_{0}\left|\Psi\left(\mathbf{r}_{i}\right)\right|^{2}$, contains the electron envelope wave function $\Psi\left(\mathbf{r}_{i}\right)$ at the nuclear site $\mathbf{r}_{i}$, and $v_{0}$ is the volume of the crystal cell. For simplicity we consider nuclear spin $1 / 2$. Neglecting dipolar interactions between the nuclei, we can consider only some particular nuclear configuration, described in the $\hat{I}_{z}^{i}$ eigenbasis as $\mid\left\{I_{z}^{i}\right\}>$, with $I_{z}^{i}= \pm 1 / 2$. 114, 15] Moreover, we assume an unpolarized configuration with a typical net nuclear magnetic field $A /\left(\sqrt{N} g \mu_{B}\right)$, being much less than $A /\left(g \mu_{B}\right)$ (fully polarized case), for the precise definition of $N$ see below. We study the decay of the electron spin from its initial $(t=0) \hat{S}_{z}$-eigenstate $\mid \Uparrow>$. For this we evaluate the correlator

$$
C_{n}(t)=<n\left|\delta \hat{S}_{z}(t) \hat{S}_{z}\right| n>
$$

where $\langle|\ldots|>$ means the diagonal matrix element. Here $\delta \hat{S}_{z}(t)=\hat{S}_{z}(t)-\hat{S}_{z}$, and $\hat{S}_{z}(t)=\exp (i t \hat{\mathcal{H}}) \hat{S}_{z} \exp (-i t \hat{\mathcal{H}})$. This correlator is proportional to $<n\left|\hat{S}_{z}(t)-\hat{S}_{z}(0)\right| n>$. Since at $t=0$ the total (electron and nuclear) state $|n>=| \Uparrow,\left\{I_{z}^{i}\right\}>$ is an eigenstate of $\hat{\mathcal{H}}_{0}=\hat{S}_{z} \hat{h}_{N z}$ (with eigenergy $\epsilon_{n}$ ), we can expand in the perturbation $\hat{V}=(1 / 2)\left(\hat{S}_{+} \hat{h}_{N-}+\hat{S}_{-} \hat{h}_{N+}\right)\left(\right.$ with $\hat{\mathcal{H}}=\hat{\mathcal{H}}_{0}+$ $\hat{V})$. Introducing the usual time evolution operator $\hat{U}(t)=\hat{T} \exp \left(-i \int_{0}^{t} d t_{1} \hat{V}\left(t_{1}\right)\right)$, with $\hat{T}$ being the timeordering operator, we get for the correlator $<n$ | $\hat{U}^{\dagger}(t) \hat{S}_{z}(t) \hat{U}(t) \hat{S}_{z}(0) \mid n>$, where the time dependence of all operators is due to the $\hat{\mathcal{H}}_{0}$ Hamiltonian. Then we obtain in leading order in $\hat{V}$,

$$
C_{n}(t)=\sum_{k} \frac{\left|V_{n k}\right|^{2}}{\omega_{n k}^{2}}\left(\cos \left(\omega_{n k} t\right)-1\right) \approx \frac{1}{\left[\epsilon_{z}+\left(h_{z}\right)_{n}\right]^{2}} \sum_{k} \frac{A_{k}^{2}}{8}\left[\cos \left(\epsilon_{z}+\left(h_{z}\right)_{n}+A_{k} / 2\right) t-1\right],
$$

where $V_{n k}=<n|\hat{V}| k>$ is the matrix element between initial state $n=\Uparrow,\left\{\ldots, I_{z}^{k}=-1 / 2, \ldots\right\}$ and intermediate state $k=\Downarrow,\left\{\ldots, I_{z}^{k}=+1 / 2, \ldots\right\}$, and $\omega_{n k}=\epsilon_{n}-\epsilon_{k}$, 
$\epsilon_{z}=g \mu_{B} B_{z}$. We have used that $\left|V_{n k}\right|^{2}=A_{k}^{2}<$ $n\left|1 / 2-\hat{I}_{z}^{k}\right| n>/ 4$, and $\omega_{n k}=\epsilon_{z}+\left(h_{z}\right)_{n}+A_{k} / 2$, where $\left(h_{z}\right)_{n}=<n\left|\hat{h}_{N z}\right| n>$, and the fact that for the typical nuclear configuration $\left(h_{z}\right)_{n}^{2} \simeq \omega_{N}^{2} \gg A_{k}^{2}$. Since $N \gg 1$, we replace the sums over $k$ (which run over the entire space) by integrals, i.e. $\sum_{k} f_{k}=\left(\int d^{3} r / v_{0}\right) f(\mathbf{r})+o(1 / N)$. Then we have

$$
C_{n}(t) \simeq-\frac{A^{2}}{8 \pi N\left(\epsilon_{z}+\left(h_{z}\right)_{n}\right)^{2}}\left[\frac{I_{0}}{2}-I_{1}(\tau) \cos \left(\epsilon_{z}+\left(h_{z}\right)_{n}\right) t+I_{2}(\tau) \sin \left(\epsilon_{z}+\left(h_{z}\right)_{n}\right) t\right]
$$

where

$I_{0}=\int_{-\infty}^{+\infty} d z \chi_{0}^{4}(z), I_{1,2}(\tau)=\int_{-\infty}^{+\infty} d z \chi_{0}^{4}(z) F_{1,2}\left(\tau \chi_{0}^{2}(z)\right)$,

and

$$
F_{1}(\eta)=\frac{\sin \eta}{\eta}+\frac{\cos \eta-1}{\eta^{2}} ; F_{2}(\eta)=\frac{\sin \eta}{\eta^{2}}-\frac{\cos \eta}{\eta} .
$$

Here $N=a_{z} a^{2} / v_{0} \gg 1$ is the number of nuclei inside the dot, and $\tau=A t / 2 \pi N$. Eq.(5) was obtained with the use of the following expression for the electron envelope wave function:

$$
|\Psi(\rho, z)|^{2}=\left(1 / \pi a^{2} a_{z}\right) \exp \left(-\rho^{2} / a^{2}\right) \chi_{0}^{2}(z) .
$$

Here $a, a_{z}$ are the dot sizes in the lateral and transverse (perpendicular to the 2D plane) directions, respectively, and the transverse wave function $\chi_{0}(z)$ is normalized, i.e. $\int_{-\infty}^{+\infty} d z \chi_{0}^{2}(z)=1$. For any analytic function $\chi_{0}^{2}(z)$ with expansion $\chi_{0}^{2}(z)=\chi_{0}^{2}(0)-$ $z^{2}\left(\chi_{0}^{2}\right)^{\prime \prime} / 2$ near its maximum, we have $I_{1,2}(\tau \gg$ $1)= \pm\left(\chi_{0}^{2}(0) / \tau^{3 / 2}\right) \sqrt{\pi /\left(\chi_{0}^{2}\right)^{\prime \prime}}\left[\sin \left(\tau \chi_{0}^{2}(0)\right) \mp \cos \left(\tau \chi_{0}^{2}(0)\right)\right]$. Thus, for this case we obtain the universal power law decay for times $\tau \gg 1$, i.e. $t \gg(A / N)^{-1}$ :

$$
\begin{array}{r}
C_{n}(\tau \gg 1) \simeq-\gamma+\frac{\tilde{\gamma}}{\tau^{3 / 2}} \sin \left(\tilde{h}_{n} t-\phi\right), \\
\tilde{h}_{n}=\epsilon_{z}+\left(h_{z}\right)_{n}+A_{0} / 2 .
\end{array}
$$

Here $A_{0}$ is the coupling constant with the nucleus located at the center of the dot; and $\phi$ is a phase shift with $\phi \sim 1$. Note that for the typical nuclear configuration the quantity $A^{2} / N\left(h_{z}\right)_{n}^{2}$ is of order unity, thus, for a weak Zeeman field $\epsilon_{z}<\omega_{N}$ the part of the electron spin state which decays is of the order of the initial value. Hence, the same holds for the spin part which survives at $\tau \gg 1$ (i.e. $\gamma \simeq \tilde{\gamma} \simeq 1 / 2$, for $\epsilon_{z}<\omega_{N}$ ). We see from Eq.(5) that in the presence of a large Zeeman field, $\epsilon_{z} \gg \omega_{N}$, the asymptotic behaviour of $C_{n}(t)$ is not changed, the only difference being that the decaying part of the initial spin state is small now, i.e. $\gamma \simeq \tilde{\gamma} \simeq\left(\omega_{N} / \epsilon_{z}\right)^{2} \ll 1$. For the fully polarized nuclear state Eqs. (5),95) should be multiplied by 2 , and $\left(h_{z}\right)_{n}$ should be replaced by $A / 2$.

We note that $C_{n}(t)$ in (4) is quasiperiodic in $t$, and, thus, it will decay only up to the Poincaré recurrence time
$\tau_{P}$. This time can be found from the condition that the terms omitted when converting sums to integrals become comparable with the integral itself. This will happen at $\tau \simeq N$, giving $\tau_{P}=0.1 s-1 s$.

In next order, $\hat{V}^{4}$, we face the problem of secular terms or "resonances", i.e. the corrections will contain zero denominators. This gives rise to linearly growing terms $\propto \omega_{N} t$, even for $t \ll(A / N)^{-1}$. In higher order the degree of the divergence will increase. This means that the decay law we found can, in principle, change after proper resummation, because no small expansion parameter exists, which, strictly speaking, would justify a perturbative approach. Still, the result found in lowest order remains qualitatively correct in that it shows that a non-uniform hyperfine coupling leads to a non-exponential decay of the spin. This conclusion is confirmed by an exactly solvable case to which we turn next.

\section{POLARIZED NUCLEI. EXACTLY SOLVABLE CASE.}

In this section we consider the exactly solvable case where the initial nuclear spin configuration is fully polarized (see Fig. 2). We also allow for a magnetic field but neglect its effect on the nuclear spins. With the initial wave function

$$
\Psi_{0}=\mid \Downarrow ; \uparrow, \uparrow, \uparrow, \ldots>
$$

we can construct the exact wave function of the system for $t>0$,

$$
\Psi(t)=\alpha(t) \Psi_{0}+\sum_{k} \beta_{k}(t) \mid \Uparrow ; \uparrow, \uparrow, \downarrow_{k}, \uparrow, \ldots>
$$

with normalization $|\alpha(t)|^{2}+\sum_{k}\left|\beta_{k}(t)\right|^{2}=1$, and we assume that $\alpha\left(t=0^{+}\right)=1, \alpha(t<0)=0$. The second term in Eq.(11) is an entangled coherent superposition of the states with exactly one nuclear spin flipped, and thus similar to a magnon excitation. The correlator $C_{0}$ is expressed through $\alpha(t)$ by the formula:

$$
C_{0}(t)=-<\Psi_{0}\left|\delta \hat{S}_{z}(t) \hat{S}_{z}\right| \Psi_{0}>=\left(1-|\alpha(t)|^{2}\right) / 2 .
$$


Then, inserting $\Psi(t)$ into the Schrödinger equation we obtain

$$
\begin{aligned}
& i \frac{d \alpha(t)}{d t}=-\frac{1}{4} A \alpha(t)+\sum_{k} \frac{A_{k}}{2} \beta_{k}(t)-\frac{\epsilon_{z} \alpha(t)}{2}, \\
& i \frac{d \beta_{l}(t)}{d t}=\left(\frac{A}{4}-\frac{A_{l}}{2}\right) \beta_{l}(t)+\frac{A_{l}}{2} \alpha(t)+\frac{\epsilon_{z} \beta_{l}(t)}{2}
\end{aligned}
$$

where $A=\sum_{k} A_{k}$. Laplace transforming (13), $\alpha(\tilde{\omega})=$ $\int_{0}^{\infty} d t \exp (-\tilde{\omega} t) \alpha(t)$, we obtain

$$
\alpha(\tilde{\omega})=\frac{i \alpha\left(t=0^{+}\right)}{i \omega+A^{\prime} / 2-\sum_{k} A_{k}^{2} / 4\left(i \omega+A_{k} / 2\right)},
$$

where $i \omega=i \tilde{\omega}-A^{\prime} / 4, A^{\prime}=A+2 \epsilon_{z}$, and we have used the fact that $\beta_{k}(t=0)=0$. Now we use the identity $\sum_{k} A_{k}^{2} / 4\left(i \omega+A_{k} / 2\right)=A / 2-i \omega \sum_{k} A_{k} / 2\left(i \omega+A_{k} / 2\right)$ and replace the sum $\sum_{k} \frac{A_{k}}{i \omega+A_{k} / 2}$ over the $x y$-plane by an integral. Calculating it using $\left|\Psi\left(\mathbf{r}_{k}\right)\right|^{2}$ given in Eq.(8) we obtain:

$$
\alpha(t)=\frac{1}{2 \pi i} \int_{\Gamma} \frac{d \omega i \exp \left[\left(\omega-i A^{\prime} / 4\right) t\right]}{i \omega+\epsilon_{z}+\pi N i \omega \int d z \ln \left(1-\frac{i A \chi_{0}^{2}(z)}{2 \pi N \omega}\right)} .
$$

As usual, the integration contour $\Gamma$ in Eq.(15) is the vertical line in the complex $\omega$-plane so that all singularities of the integrand lie to its left (see Fig. 3). These singularities are: two branch points $\left(\omega=0, \omega_{0}=i A \chi_{0}^{2}(0) / 2 \pi N \equiv\right.$ $i A_{0} / 2$ ), and first order poles which lie on the imaginary axis $(\omega=i v)$. For $\epsilon_{z}>0$ there is one pole, while for $\epsilon_{z}<0$ there are two poles, and for $\epsilon_{z}=0$ there is one first order pole at $\omega_{1} \approx i A / 2+i A \int d z \chi_{0}^{4}(z) / 4 \pi N$. For the contribution from the branch cut between $\omega=0$ and $\omega=\omega_{0}$ we obtain

$$
\begin{gathered}
\tilde{\alpha}(t)=\frac{e^{-i A^{\prime} t / 4}}{\pi N} \int_{0}^{1} d \kappa 2 z_{0} \kappa e^{i \tau^{\prime} \kappa}\left\{\left[\kappa \int d z \ln \left|1-\frac{\chi_{0}^{2}(z)}{\chi_{0}^{2}(0) \kappa}\right|\right.\right. \\
\left.\left.+\quad \kappa / \pi N-2 \epsilon_{z} / A \chi_{0}^{2}(0)\right]^{2}+\left(2 \pi z_{0}\right)^{2} \kappa^{2}\right\}^{-1},
\end{gathered}
$$

where $\tau^{\prime}=\tau \chi_{0}^{2}(0)$, and $z_{0}=z_{0}(\kappa)$ is defined through $\chi_{0}^{2}\left(z_{0}\right)=\chi_{0}^{2}(0) \kappa$. We have introduced the dimensionless variable $\kappa=\omega / \omega_{0} \leq 1$. In terms of this new variable the argument of the log function in Eq.(15) has the form $1-\chi_{0}^{2}(z) / \kappa \chi_{0}^{2}(0)$. Therefore, for a given $\kappa$ this expression changes the sign at $z_{0}(\kappa)$ which can be found from the equation given above.

Thus, the physical picture can be described as follows. At the initial time $t=0$ the system has some energy corresponding to the pole and starts to oscillate back and forth each time visiting different frequencies within the branch cut which corresponds to the flip-flop processes with the nuclei located at different sites. Therefore, the contribution from the branch cut describes the electron spin decoherence. At $\tau$ of the order of unity (where the decay mainly occurs) the decoherence is due to the interaction with the nuclei located at distances of the order of the dot radius where the derivative of the coupling constant is maximal. For longer times, $\tau \gg 1$, the asymptotics is determined either by the interaction with the nuclei located far from the dot or near the dot center depending on the Zeeman field value.

First we consider the case $\epsilon_{z}=0$, i.e. no magnetic field. The asymptotic behavior of Eq.(16) for $\tau \gg 1$ is determined by $\kappa \ll 1$. For example, for $\chi_{0}^{2}(z) / \chi_{0}^{2}(0)=$ $\exp \left(-z^{2}\right)$ we find

$$
\tilde{\alpha} \propto 1 / \ln ^{3 / 2} \tau .
$$

This behaviour is not universal and is determined by the form of the electron wave function at distances that are large compared to the dot size since at large $\tau$ the decoherence is due to the interaction with the nuclei located far from the dot. As a result, a nuclear spin diffusion induced by the hyperfine interaction occurs in that region.

Thus, the decay of $|\alpha(t)|$ starts at $\tau>1$, i.e. at $t>N / A$, as in the unpolarized case (see Fig. 4). Note that the magnitude of $\tilde{\alpha}$ is of order of $1 / N$ (see also Ref.16), thus, the decaying part of the initial spin state has this smallness as well, in contrast to the unpolarized case above where this part is of order one. The reason for this smallness is that for a fully polarized state the gap seen by the electron spin through the hyperfine interaction is $A / 2$, therefore, only a small portion $\sim 1 / N$ of the opposite $(+1 / 2)$ state can be admixed. Indeed, in this case the change of the energy of the electron subsystem is $\sim A / N$ which can be compensated by the energy (order of $A / N)$ of a magnon excited in the nuclear subsystem.

For a large Zeeman field $\left(\left|\epsilon_{z}\right| \gg A\right)$ and for $\tau \gg 1$, the main contribution in (16) is given for $\kappa \rightarrow 1$, i.e. by the interaction with the nuclei located near the dot center. Expanding $\chi_{0}^{2}(z)$ for small $z$ (see above), we obtain $z_{0}^{2}=$ $2 \chi_{0}^{2}(0)(1-\kappa) /\left(\chi_{0}^{2}\right)^{\prime \prime}$. Then from Eq.116) we have for $\left|\epsilon_{z}\right| \gg A$

$$
\tilde{\alpha}(\tau \gg 1)=\frac{-e^{i \tau^{\prime}-i A^{\prime} t / 4}}{4 \sqrt{\pi} N} \frac{\chi_{0}^{2}(0)}{\sqrt{\left(\chi_{0}^{2}\right)^{\prime \prime}}} \frac{A^{2}}{\epsilon_{z}^{2}} \frac{(1+i)}{\tau^{3 / 2}} .
$$

From this we find then that the correlator (12) agrees with the perturbative result (5) for the fully polarized state, i.e. $C_{0}(t)-C_{0}(\infty) \propto 1 / t^{3 / 2}$. (Note that the asymptotic behaviour of the correlator is given by the term which is the cross product of the pole contribution and the one (Eq.(18)) from the branch cut.) This agreement is to be expected, since for a large Zeeman field, the perturbative treatment with a small parameter $A /\left|\epsilon_{z}\right| \ll 1$ is meaningful (the same is true for any model with a small expansion parameter, for example, for a system with anisotropy, where the hyperfine constants in perpendicular and transverse directions are different, see section III B). However, at zero Zeeman field, when the system cannot be treated perturbatively, we find $C_{0}(t)-C_{0}(\infty) \propto 1 / \ln ^{3 / 2} t$, and the agreement with (5) breaks down. Nevertheless, the characteristic time scale for the onset of the non-exponential decay is the same for all cases and given by $(A / N)^{-1}$. We have also 
checked that $<\hat{S}_{x}(t)-\hat{S}_{x}(0)>$ has the same behaviour as $<\hat{S}_{z}(t)-\hat{S}_{z}(0)>$ with the same characteristic time $\mathrm{N} / \mathrm{A}$.

Finally we mention that the observed decay of the electron spin can be experimentally studied by local NMR measurements [17.

\section{A. Fully polarized nuclei. 2D case}

Here we consider the $2 \mathrm{D}$ case when there is no variation of the coupling constants in one direction, i.e. we use the model representation $\chi_{0}^{2}(z)=\theta(1 / 2-|z|)$. This case allows us to follow the dependence of the electron spin decay law on the spatial variation of the electron wave function in different directions. From Eq.(15) we obtain the following singularities: two branch points $\left(\omega=0, \omega_{0}=i A / 2 \pi N\right)$ and the first order poles which lie on the imaginary axis $(\omega=i v)$. The position of these poles can be found from the equation:

$$
\exp \left(-\frac{1}{\pi N}\right) \exp \left(\frac{2 \epsilon_{z} \xi}{A}\right)=1-\xi ; \xi=A / 2 \pi N v
$$

For positive $\epsilon_{z}$ there is only one solution of this equation, for negative $\epsilon_{z}$ - there are two. Thus, at $\epsilon_{z}=0$ there is one first order pole at $\omega_{1}=\frac{i A}{2 \pi N} \frac{1}{[1-\exp (-1 / \pi N)]}$. For the contribution from the branch cut between $\omega=0$ and $\omega=\omega_{0}$ we obtain:

$$
\tilde{\alpha}(t)=\frac{\exp \left(-i A^{\prime} t / 4\right)}{\pi N} \int_{0}^{1} \frac{d \kappa \kappa \exp (i \tau \kappa)}{\left[\kappa \ln (-1+1 / \kappa)+(\kappa / \pi N)-\left(2 \epsilon_{z} / A\right)\right]^{2}+\pi^{2} \kappa^{2}} .
$$

Let us consider first the case of zero Zeeman field. Then we have $\alpha(t>0)=$ $\exp (-i A t / 4) \exp \left(\omega_{1} t\right) /(\pi N[\exp (1 / \pi N)-1])+\tilde{\alpha}(t)$. The asymptotic behaviour of Eq.(20) at $\epsilon_{z}=0$ and for $\tau \gg 1$ is $1 / \ln \tau$. In the case of a strong Zeeman field, $\left|\epsilon_{z}\right| \gg A$, it follows from Eq.(20) that the asymptotics at $\tau \gg 1$ is $1 / \tau$. Note, that this asymptotics is true in the interval $1 \ll \tau \ll \exp \left(2\left|\epsilon_{z}\right| / A\right)$. Then we obtain:

$$
<\hat{S}_{z}(t)>=\frac{1}{2}-|\alpha|^{2}=-\frac{1}{2}\left[1-\frac{A^{2}}{2 \pi N \epsilon_{z}^{2}}\right]-\frac{A^{2}}{2 \pi N \epsilon_{z}^{2}} R e\left\{\frac{\exp \left(-i A^{\prime} t / 2\right) \exp (i \tau)}{i \tau}\right\}
$$

The change of the asymptotics occurs even at $\left|\epsilon_{z}\right| \ll A$. If $1 \ll \tau / \ln \tau \ll A /\left|\epsilon_{z}\right|$, then the asymptotics is as before, i.e. $1 / \ln \tau$. For $\tau / \ln \tau \gg A /\left|\epsilon_{z}\right| \gg 1$ it is $1 /\left(\tau \ln ^{2} \tau\right)$.

\section{B. Anisotropy in the exactly solvable model.}

We consider here the model where the hyperfine constants in the $z$-direction and in the transverse direction are different (there is no Zeeman field): $A_{i}^{z}=A_{z} v_{0}$ $\left.\Psi\left(\mathbf{r}_{i}\right)\right|^{2}, A_{i}^{\perp}=A_{\perp} v_{0}\left|\Psi\left(\mathbf{r}_{i}\right)\right|^{2}$. Then from a system of the equations similar to Eq.(13) we obtain the following solution (again for the $2 \mathrm{D}$ case, when $\chi_{0}^{2}(z)=$ $\theta(1 / 2-|z|))$ :

$$
\alpha(t)=\frac{i}{2 \pi i} \int_{\Gamma} d \omega \frac{\exp \left(-i A_{z} t / 4\right) \exp (\omega t)}{i \omega+\left(A_{z} / 2\right)-\left(A_{\perp}^{2} / 2 A_{z}\right)+\pi N\left(A_{\perp}^{2} / A_{z}^{2}\right) i \omega \ln \left(1-\frac{i A_{z}}{2 \pi N \omega}\right)} .
$$

Note that this equation has the same form as Eq.(15), i.e. an anisotropy plays the role of a Zeeman field. Then for the contribution from the branch cut (the decaying part of the initial spin state) we have: 


$$
\tilde{\alpha}(t)=\frac{\exp \left(-i A_{z} t / 4\right)}{\pi N \sigma} \int_{0}^{1} \frac{d \kappa \kappa \exp (i \tau \kappa)}{[\kappa \ln (-1+1 / \kappa)+(\kappa / \pi N \sigma)+1-1 / \sigma]^{2}+\pi^{2} \kappa^{2}}
$$

where $\sigma=A_{\perp}^{2} / A_{z}^{2}$, and $\tau$ is defined now as $\tau=A_{z} t / 2 \pi N$. In the case of strong anisotropy, $\sigma \ll 1$, we have at $\tau \gg 1$ :

$$
<\hat{S}_{z}(t)>=\frac{1}{2}-|\alpha|^{2}=-\frac{1}{2}\left(1-\frac{2 \sigma}{\pi N}\right)-\frac{2 \sigma}{\pi N} \operatorname{Re}\left\{\frac{\exp \left(-i A_{z} t / 2\right) \exp (i \tau)}{i \tau}\right\} .
$$

The same result follows for the polarized state from Eq.(倢) in the perturbative approach.

\section{Some features of the fully polarized state.}

There are several interesting features which we can observe for the fully polarized state. In an external Zeeman field, the effective gap seen by the electron spin is $A^{\prime} / 2=A / 2+\epsilon_{z}$. Thus, when $\epsilon_{z}$ is made negative this gap decreases and even vanishes at $\left|\epsilon_{z}\right|=A / 2$. From Eq.115) we find that the two poles are symmetric in this case, and the system resonates between the two frequencies $\omega_{ \pm}= \pm i A\left(\int \chi_{0}^{4}(z) d z\right)^{1 / 2} / \sqrt{8 \pi N}$. Note that the residual gap is of order $A / \sqrt{N}$ (and not $A / N$, as one might naively expect). Near this Zeeman field we have $|\alpha(t)|^{2}=\cos ^{2}\left(\omega_{+} t\right)$ (up to small corrections of order $1 / N)$, and, as a result, $|\alpha|^{2}$ averaged over time is $1 / 2$, i.e. the up and down states of the electron spin are strongly coupled via the nuclei (see Fig. 5). In contrast, outside this resonance regime the value of $|\alpha|^{2}$ is close to 1 (again with small $1 / N$ corrections), i.e. $\left\langle\hat{S}_{z}(t)>=1 / 2-|\alpha|^{2}\right.$ is close to $-1 / 2$ at any time. The width of the resonance is $\sim A / \sqrt{N}$, i.e. small compared to the initial gap $A / 2$. We note that this behavior represents periodic (Rabi) oscillations with a single well-defined frequency and is not related to decoherence. [The latter is described by the branch cut contribution $\tilde{\alpha}$ which remains small (order $1 / \mathrm{N}$ ) even near the resonance.] This abrupt change in the amplitude of oscillations of $<\hat{S}_{z}(t)>$ (when changing $\epsilon_{z}$ in a narrow interval around $A / 2$ ) can be used for an experimental detection of the fully polarized state. Note that the weight of the upper pole alone (i.e. that which exists at $\epsilon_{z}=0$ ) also drops abruptly from a value close to 1 to a value much smaller than 1 in the same narrow interval, which can be experimentally checked by Fourier analysis.

Another special value of the Zeeman field corresponds to the case when the upper pole is close to $\omega_{0}(\kappa=1)-$ the upper edge of the branch cut. This occurs (see
Eq.(15)) at the critical value $\epsilon_{z}^{\star}=b A / 2<0$, where $b=$ $\chi_{0}^{2}(0) \int d z \ln \left|1-\chi_{0}^{2}(z) / \chi_{0}^{2}(0)\right|<-1$ is a non-universal number which depends on the dot shape. Since at a finite Zeeman field the asymptotics in $t$ is determined by $\kappa$ 's close to 1 , we see from Eq.(16) that for $\epsilon \approx \epsilon_{z}^{\star}$ the asymptotics changes abruptly. Indeed, for $\left(\left(\epsilon_{z}-\epsilon_{z}^{\star}\right) / A\right)^{2} \ll 1$, we find $\tilde{\alpha} \propto 1 / \sqrt{\tau}$, for $1 \ll \tau \ll\left(\left(\epsilon_{z}-\epsilon_{z}^{\star}\right) / A\right)^{-2}$, and $\tilde{\alpha} \propto 1 / \tau^{3 / 2}$, for $\tau \gg\left(\left(\epsilon_{z}-\epsilon_{z}^{\star}\right) / A\right)^{-2}$. Thus, when approaching the critical Zeeman field $\epsilon_{z}^{\star}$ there is a slow down of the asymptotics from $1 / \tau^{3 / 2}$ to $1 / \tau^{1 / 2}$. It is interesting that this slow down is related to a strong modulation of the density of states (DOS) of the excitations within the continuum band (branch cut) near its edge when $\epsilon_{z} \rightarrow \epsilon_{z}^{\star}$. In the subspace of none or one nuclear spin flipped (see Eq.(11)), the DOS $\nu$ becomes

$$
\nu(u)=\frac{1}{\pi} \operatorname{Im}\left[G_{0}(u)+\frac{d}{d u} \ln D(u)\right]
$$

where $u=i \omega, G_{0}(u)=\sum_{k} 1 /\left(u+A_{k} / 2\right)$ is the "unperturbed Green's function", and $D(u)$ is the denominator of $\alpha(\omega)$, see Eq.(14). [20] The derivation of Eq.(25) is given in the Appendix. One can then show that for $\epsilon_{z} \rightarrow \epsilon_{z}^{\star}$ (i.e. the upper pole approaches the continuum edge), the DOS develops a square root singularity: $\nu(u) \propto 1 / \sqrt{\omega_{0}-u}$. Simultaneously, the weight of the upper pole vanishes linearly in $\epsilon_{z}$ as $\epsilon_{z}^{\star}-\epsilon_{z} \rightarrow 0$.

Finally, the nuclear state is characterized by $\beta_{k}(t)$, which allows for similar evaluation as for $\alpha$. Here we just note that its branch cut part, $\tilde{\beta}_{k}(t)$, is nonmonotonic in time, particularly pronounced at $\epsilon_{z} \rightarrow \epsilon_{z}^{\star}$ : First, $\tilde{\beta}_{k}(t)$ grows like $\sqrt{\tau}$, until $\tau$ reaches $\sim 1 /\left(1-a_{k}\right) \gg 1$, and then it decays like $1 /\left(\sqrt{\tau}\left(1-a_{k}\right)\right)$, with $a_{k}=A_{k} / A_{0} \rightarrow 1$. Thus, $\beta_{k}$ is maximal for $A_{k}$ close to $A_{0}$, i.e. the nuclei near the dot-center are affected most by the hyperfine interaction with the electron spin. 


\section{DEPENDENCE OF THE ELECTRON SPIN DECOHERENCE ON THE INITIAL NUCLEAR STATE.}

So far we have assumed that the initial nuclear state had the form of a single tensor product state. Now we study the dependence of the electron spin decoherence on the initial nuclear state and start with the simple case of homogeneous coupling.

\section{A. Homogeneous coupling.}

In this subsection we consider the case of homogeneous coupling, when all the coupling constants are equal, i.e. $A_{k}=A / N$. It is instructive to start with it, since in contrast to naive expectations even in this simple case there is some time dependence of $\left\langle S_{z}(t)\right\rangle$ which cannot be described by a single frequency. On the other hand, in this case the expectation value $\left\langle S_{z}(t)>\right.$ is a periodic function and, for that reason, the electron spin dynamics is coherent though the corresponding period can be very large for large $N$ (see below). The Hamiltonian is very simple, $\hat{H}=(A / N) \mathbf{I} \cdot \mathbf{S}$, where $\mathbf{I}$ is the total nuclear spin. Then, we can diagonalize $\hat{H}$ and obtain the result:

$$
\begin{array}{r}
E_{1}=\frac{A I}{2 N}, \text { for } J_{1}=I+1 / 2, \\
E_{2}=-\frac{A(I+1)}{2 N}, \text { for } J_{2}=I-1 / 2 .
\end{array}
$$

In the simplest case of a fully polarized nuclear state and opposite initial electron spin polarization (with $\Psi_{0}$ given in Eq.(10)) we obtain the following result:

$$
<S_{z}(t)>=-\frac{1}{2}+\frac{2 N}{(N+1)^{2}}\left(1-\cos \Omega_{N / 2} t\right)
$$

where $\Omega_{N / 2}=A(1+1 / N) / 2$. This result can be easily understood, since for the homogeneous coupling $I^{2}$ is conserved and the initial nuclear state corresponds to the maximal value $I=N / 2$. Then from Eq. 26) we obtain for the difference of $E_{1}$ and $E_{2}$ corresponding to $I=N / 2$ the value which is equal to $\Omega_{N / 2}$. Note that the magnitude of the oscillating term is $1 / N$ for $N \gg 1$, as it has been already observed in Sec. III.

In the case when initially one nuclear spin is flipped, the exact ket has the form

$$
\begin{array}{r}
\Psi_{1}(t)=\sum_{k} \alpha_{k}(t) \mid \Downarrow ; \uparrow, \uparrow, \downarrow_{k}, \uparrow, \ldots>+ \\
+\sum_{k>l} d_{k l}(t) \mid \Uparrow ; \uparrow, \uparrow, \downarrow_{k}, \uparrow, \downarrow_{l}, \ldots>
\end{array}
$$

and the normalization condition is $\sum_{k}\left|\alpha_{k}(t)\right|^{2}+$ $\sum_{k>l}\left|d_{k l}(t)\right|^{2}=1$, with initial condition $d_{k l}(0)=0$. From the solution of the Schrödinger equation we obtain for $<S_{z}(t)>$

$$
<S_{z}(t)>=-\frac{1}{2}+\frac{2(N-2)}{(N-1)^{2}}\left(1-\cos \Omega_{N / 2-1} t\right)-\frac{2|\alpha(0)|^{2}}{N}\left[\frac{(N-2)}{(N-1)^{2}}\left(1-\cos \Omega_{N / 2-1} t\right)-\frac{2(N-1)}{(N+1)^{2}}\left(1-\cos \Omega_{N / 2} t\right)\right]
$$

where $\Omega_{N / 2-1}=A(1-1 / N) / 2$ is the frequency corresponding to the solution $E_{1}-E_{2}$ of Eq.(26) with $I=N / 2-1$, and $\alpha(0)=\sum_{k} \alpha_{k}(0)$. Now, in contrast to the fully polarized case there are oscillating terms with two frequencies involved. This is because the initial nuclear state corresponds to $I_{z}=N / 2-1$. This value of $I_{z}$ can be realized with $I=N / 2$ and $I=N / 2-1$. Note that for $N \gg 1$ the amplitude of the oscillating term corresponding to $I=N / 2$ is smaller by factor $1 / N$ than the amplitude of the oscillating term corresponding to $I=N / 2-1$. This is a general rule which can be checked for an arbitrary initial state with $I_{z}=n$. This state is constructed mostly from the state with $I=n$ and the contributions of all other $I$ values are small for $N \gg 1$. The expectation value $\left\langle S_{z}(t)\right\rangle$, Eq.(29), is periodic with period $T=4 \pi N / A$. The initial condition enters Eq.(29) through the quantity $\alpha(0)=\sum_{k} \alpha_{k}(0)$. We can easily check that in the case of the homogeneous coupling there is only a weak dependence on the type of the initial nuclear state. For example, the cases $\alpha_{k}(0)=\delta_{k n}, \alpha(0)=1$ (which is a single tensor product state) and a randomly correlated (entangled) state when the coefficients $\alpha_{k}(0)$ have random phases, so that $|\alpha(0)| \ll \sqrt{N}$, correspond to the same solution $<S_{z}(t)>$ for $N \gg 1$, as is easily seen from Eq.(29).

The solution for the initial nuclear state with a larger number of flipped spins can be obtained similarly. For example, when the initial state corresponds to two flipped nuclear spins, the solution for $\left\langle S_{z}(t)\right\rangle$ contains oscillating terms with three frequencies, $\Omega_{N / 2}, \Omega_{N / 2-1}, \Omega_{N / 2-2}$. These frequencies are the solutions of Eq.(26) for $I=$ $N / 2, N / 2-1, N / 2-2$. Moreover, the initial conditions (the information about the initial nuclear state) enter now $<S_{z}(t)>$ in a more complicated way besides the quantity $|\alpha(0)|^{2}$ which we observed for the case with one flipped spin, since it contains also the quantity $\sum_{k}\left|\alpha_{k}(0)\right|^{2}$. Again, depending on the type of the correlations between the coefficients $\alpha_{k}(0)$ we can have 
different dynamics of the electron spin. However, the dependence on the type of the initial nuclear state is again rather weak.

\section{B. Inhomogeneous coupling.}

Up to now we have only considered the decoherence of the electron spin caused by inhomogeneous coupling for a given initial nuclear state which had the form of a single tensor product state. Here we consider a more general initial nuclear state which can be entangled, i.e. contains the coherent superposition of the single tensor product states $\sum_{T} \alpha_{T}|T\rangle$, where the sum goes over the tensor product states $|T\rangle$. This problem was addressed numerically in Ref.19. We give here the exact analytical solution of a typical problem of this kind which consists of a very simple initial nuclear state but still contains the relevant physics. In particular, we can examine the dependence of the electron spin decoherence (and the corresponding time scales) on the type of initial state. As an example of the initial electron and nuclear state which we can treat exactly we choose the following one: All but one nucleus are polarized in z-direction and the electron spin is in the up state. We will see that the results are the same for all cases, namely, for this particular nuclear state the time scale for the onset of the decay is always $\sim N / A$ independent of the phases of the coefficients $\alpha_{T}$.

The exact ket of the system for $t>0$ has the form

$$
\Psi(t)=\sum_{k} \alpha_{k}(t)\left|\Uparrow ; \uparrow, \uparrow, \downarrow_{k}, \uparrow, \ldots>+\beta(t)\right| \Downarrow ; \uparrow, \uparrow, \uparrow, \ldots>
$$

where the first term at $t=0$ represents the initial state of the system. The normalization condition is $\sum_{k}\left|\alpha_{k}(t)\right|^{2}+|\beta(t)|^{2}=1$, and $\beta(t=0)=0$. The dependence on the initial nuclear state comes from a different realization of the coefficients $\alpha_{k}(0)$. If, for example, we have $\alpha_{k}(0)=\delta_{k n}$, then the initial nuclear state consists of a single product state. On the other hand, the entanglement of the initial nuclear state can be of a different kind, depending on whether the coefficients $\alpha_{k}(0)$ have random phases or correlated phases. From the system of equations similar to Eq.(13) we obtain:

$$
\begin{gathered}
\beta(t)=e^{-i t A / 4} \int_{\Gamma} \frac{d \omega}{2 \pi i} \exp (\omega t) \beta(\omega) \\
\beta(\omega)=\frac{i}{2} \frac{1}{i \omega+A / 2-\sum_{i} A_{i}^{2} / 4\left(i \omega+A_{i} / 2\right)} \sum_{k} \frac{A_{k} \alpha_{k}(0)}{i \omega+A_{k} / 2} .
\end{gathered}
$$

Here again $A=\sum_{k} A_{k}$ and the integration contour $\Gamma$ is the same as in Eq.(15), see Fig. 3. The quantity < $\hat{S}_{z}(t)>$ we are interested in is given by the equation

$$
<\hat{S}_{z}(t)>=\frac{1}{2}-|\beta(t)|^{2}
$$

As it was already mentioned above, Eq.(32) contains the information about the initial nuclear state through the coefficients $\alpha_{k}(0)$. Note that the denominator of the first factor in the solution for $\beta(\omega)$, see Eq. 32 ), is exactly the same as that in $\alpha(\omega)$, Eq.(14), if we put in the latter $\epsilon_{z}=0$. Thus, Eq. (32) contains partially the same singularities as $\alpha(\omega)$. There are, however, some additional singularities whose character depends on the properties of the coefficients $\alpha_{k}(0)$.

1. Let us consider the case $\alpha_{k}(0)=\delta_{k n}$. This means that the initial nuclear state is just a simple tensor product state. Then besides the branch cut and the first order pole (outside the branch cut) which we had before for $\alpha(\omega)$, there is an additional first order pole $\omega=i A_{n} / 2$ which lies within the branch cut. Considering again the continuous limit (i.e. replacing the sum by an integral and using Eq.(8)) we obtain:

$$
\beta_{n}(t)=\frac{e^{-i t A / 4}}{4 \pi i} \int_{\Gamma} \frac{d \omega}{\omega} e^{\omega t} \frac{A_{n}}{\left(i \omega+A_{n} / 2\right)} \frac{1}{\left[1+\pi N \int d z \ln \left(1-\frac{i A \chi_{0}^{2}(z)}{2 \pi N \omega}\right)\right]}
$$

We have evaluated Eq.(34) in the 2D case with the model

function $\chi_{0}^{2}(z)$ introduced in Sec. III A and find

$$
\beta_{n}(\tau)=e^{-i t A / 4} \frac{x_{0}}{\pi N}\left[P \int_{0}^{1} \frac{d x e^{i x \tau}}{x\left(x_{0}-x\right)\left[\pi^{2}+\ln ^{2}(1 / x-1)\right]}-e^{i \pi N \tau+i \tau / 2}-\frac{\ln \left(1 / x_{0}-1\right) e^{i x_{0} \tau}}{x_{0}\left[\pi^{2}+\ln ^{2}\left(1 / x_{0}-1\right)\right]}\right]
$$


from Eq.(33) that the decaying part of the electron spin state has now a smallness $\sim 1 / N^{2}$. This is simply due to the fact that initially the electron has the same spin orientation as all the nuclei except one, and the flip-flop process is only allowed with this particular nucleus. However, the time scale for the onset of the decoherence is the same as before, i.e. it starts at $\tau>1$ and the asymptotic dependence of Eq.(33) at $\tau \gg 1$ is $1 / \ln \tau$, the same as before, see Sec. III A.

2. Let us now consider the case when $\alpha_{k}(0)=1 / \sqrt{N}$. This corresponds to the entangled initial nuclear state where all the terms have the same phases. It is easy to see that the singularity of the second factor in Eq.(32) (a branch cut) coincides with that of the first factor. Thus, we obtain that the decay starts at the same time $\tau>1$ as it was for the single tensor product state with the same decay law, i.e. $1 / \ln \tau$.

3. Finally, we consider the case when the phases of the coefficients $\alpha_{k}(0)$ are random. It is obvious that while the decay law can be different depending on the particular choice of the coefficients $\alpha_{k}(0)$ (phases), the characteristic time scale for the onset of the decay is always the same, i.e. $N / A$. This follows from the fact that the singularity of the first factor in Eq.(32) (a branch cut) is exactly the same as of the second factor. This in turn is the consequence of the fact that in the model considered here it is only one nuclear spin which is flipped. Therefore, the characteristic energy change which determines the time scale for the onset of the decay is $A_{k} \simeq A / N$. It is also clear that in a more general case, for example, of an unpolarized nuclear state there is a different energy scale involved which is related to different values of the magnetic fields corresponding to different configurations which are present in the initial superposition of the states. This scale presumably is $A / \sqrt{N}$. Then the result can be entirely different. It has actually been observed in Ref. 19 that there is a strong dependence of the characteristic time scale for the onset of the electron spin decay on the type of the initial nuclear spin state.

\section{AVERAGING OVER NUCLEAR CONFIGURATIONS. DEPHASING TIME FOR AN ENSEMBLE OF DOTS.}

In Secs. II and III we have seen that the decay of $C_{n}(t)$ occurs in the time interval $N / A \ll t \ll N^{2} / A$, with $N / A \simeq 10^{-6} s$ in GaAs dots. On the other hand, the electron spin precesses in the net nuclear field (see Eq. (5)) with the characteristic period $\left(h_{z}\right)_{n}^{-1} \simeq \omega_{N}^{-1} \simeq$ $10^{-8} \div 10^{-9} s$. Thus, $\omega_{N}^{-1} \ll N / A$, and we see that the electron spin undergoes many precessions in a given nuclear field before decoherence sets in due to the nonuniform hyperfine couplings $A_{k}$. This behavior changes dramatically when we average over nuclear configurations 110, 21. For that purpose we consider high temperatures, $k_{B} T \gg \hbar \omega_{N}$, and average $C_{n}(t)$ in Eq. (㺼) over all nuclear configurations, i.e. $C(t)=\sum_{n} C_{n}(t) / \sum_{n}$. We then find

$$
C(t)=\sum_{k} \frac{-A_{k}^{2}}{8} \int_{0}^{t} d t_{1} \int_{0}^{t} d t_{2} \prod_{i \neq k} \cos \left[\frac{A_{i}}{2}\left(t_{1}-t_{2}\right)\right] .
$$

For $\tau \ll 1$, we get $\prod_{i \neq k} \cos \left(A_{i} t / 2\right)=$ $\exp \left[-N C(A t / 2 \pi N)^{2}\right]$, where $C \stackrel{1}{=} \pi \int d z \chi_{0}^{4}(z) / 4$. Thus, the averaged spin correlator $C(t)$, Eq.(36), is of order $-\int_{0}^{\omega_{N} t} d x \Phi(x)$, with $\Phi$ being the error function. Thus, $C(t)$ grows without bound as $\omega_{N} t$ for $\omega_{N} t \gg 1$ (the condition $\tau \ll 1$ can still be satisfied). Consequently, the perturbative approach breaks down even in leading order in $\hat{V}$ (we recall that without averaging the divergences occur in all higher but not in lowest order). To treat this case properly, we need a non-perturbative approach. For that purpose we calculate now the correlator $C(t)$ exactly by treating the nuclear field purely classically, i.e. as a c-number. Then we obtain,

$$
C_{n}(t)=-\frac{h_{N \perp}^{2}}{4 h_{N}^{2}}\left(1-\cos h_{N} t\right),
$$

where $h_{N}=\sqrt{h_{N z}^{2}+h_{N \perp}^{2}}$ is the nuclear field, with $h_{N \perp}^{2}=h_{N x}^{2}+h_{N y}^{2}$. The value of $h_{N}$ corresponds to a given nuclear configuration $n$. To make contact with the perturbation procedure we used before in the quantum case we go to the regime $h_{N}^{2} \ll h_{N z}^{2}$, where $h_{N}$ can be replaced by $h_{N z}$ in Eq.(37). Then we average the resulting expression $\left(h_{N \perp}^{2} / h_{N z}^{2}\right)\left(1-\cos h_{N z} t\right)$ over a Gaussian distribution for $h_{N}$, i.e. over $P\left(h_{N}\right) \propto$ $\exp \left(-3 h_{N}^{2} / 2 \omega_{N}^{2}\right)$. The result becomes proportional to $\int_{0}^{+\infty} d z \exp \left(-z^{2} / 2\right)(1-\cos (\gamma z)) / z^{2} \propto \int_{0}^{\gamma} d x \Phi(x)$, where $\gamma=\omega_{N} t / \sqrt{3}$. Thus, we see that we obtain exactly the same functional form as before from Eq.(36) with the same divergencies in $t$. This reassures us that the treatment of the nuclear field as a classical field is not essential. On the other hand, the same Gaussian averaging procedure can now be applied to the non-perturbative form Eq.(37). Defining $C_{c l}(t)=\int d h_{N} P\left(h_{N}\right) C_{n}(t)$, we obtain

$$
C_{c l}(t)=-\frac{1}{6}\left[1+\left(\frac{\omega_{N}^{2} t^{2}}{3}-1\right) e^{-\omega_{N}^{2} t^{2} / 6}\right] .
$$

Thus we get rapid (Gaussian) decay of the correlator for $t \gg \omega_{N}^{-1}$, giving the dephasing time $\omega_{N}^{-1}=\sqrt{N} / A$. This means that $\left\langle\hat{S}_{z}(t) S_{z}\right\rangle$ saturates at $1 / 3$ of its initial value of $1 / 4$. Finally, it seems likely that for the case of nuclear quantum spins a non-perturbative treatment of the averaged correlator $C(t)$ will lead to a similar rapid time decay as found for the classical case in Eq. (38).

\section{CONCLUSION.}

In conclusion, we have studied the spin decoherence of an electron confined to a single quantum dot in the presence of hyperfine interaction with nuclear spins. The decoherence is due to a non-uniform coupling of the electron 
spin to nuclei located at different sites. The decoherence time is given by $\hbar N / A$ and is of the order of several $\mu s$ for typical GaAs dots. It is shown that in a weak external Zeeman field the perturbative treatment of the electron spin decoherence is impossible, in particular, we cannot use the usual formulas Eq.(1). Moreover, the decay of the electron spin correlator in time does not have an exponential character, instead it is given by a power or inverse logarithm law. In the case of a strong Zeeman field the decay has a universal character $\propto 1 / t^{d / 2}$, where $d$ is the real space dimensionality of the problem. We have also solved exactly several model problems which allowed us to investigate the dependence of the electron spin decay on the initial state of the nuclear system. We have shown that there is a strong difference between the decoherence time for a single dot, $\hbar N / A$, and the dephasing time for an ensemble of dots, $\hbar \sqrt{N} / A$.

\section{ACKNOWLEDGEMENTS.}

We acknowledge support from the NCCR Nanoscience, Swiss NSF, US DARPA and ARO. Part of this work was performed at the Aspen Center of Physics and at the ITP, UC Santa Barbara. L.G. acknowledges support from NSF Grant No. DMR-9731756. We thank V. Golovach, J. Schliemann, Al.L. Efros, H. Gassmann, and F. Marquardt for useful discussions.

\section{APPENDIX}

In this Appendix we derive the density of states (DOS) given in Eq. 25). The definition for the DOS is

$$
\nu(\tilde{\omega})=\frac{1}{\pi} \operatorname{Im} \operatorname{Tr} \frac{1}{i \tilde{\omega}-\hat{\mathcal{H}}}, \quad i \tilde{\omega}=E-i \epsilon, \quad \epsilon \rightarrow 0 .
$$

We write the trace as follows:

$\operatorname{Tr} \frac{1}{i \tilde{\omega}-\hat{\mathcal{H}}}=<\Psi_{0}\left|\frac{1}{i \tilde{\omega}-\hat{\mathcal{H}}}\right| \Psi_{0}>+\sum_{k}<\Psi_{k}\left|\frac{1}{i \tilde{\omega}-\hat{\mathcal{H}}}\right| \Psi_{k}>$,

where

$$
\begin{array}{r}
\left|\Psi_{0}>=\right| \Downarrow ; \uparrow, \uparrow, \uparrow, \ldots>, \\
\left|\Psi_{k}>=\right| \uparrow ; \uparrow, \uparrow, \downarrow_{k}, \uparrow, \ldots>, k=1, \ldots, N .
\end{array}
$$

The Laplace transform of Eq. 13), $\alpha(\tilde{\omega})=$ $\int_{0}^{\infty} d t \exp (-\tilde{\omega} t) \alpha(t)$, gives for general initial condi- tions:

$$
\beta_{l}(\tilde{\omega})=\frac{A_{l} \alpha(\tilde{\omega}) / 2+i \beta_{l}(t=0)}{i \tilde{\omega}-A^{\prime} / 4+A_{l} / 2}
$$

and

$$
\alpha(\tilde{\omega})=\frac{i \alpha(t=0)}{D(\tilde{\omega})}+\frac{i}{2 D(\tilde{\omega})} \sum_{k} \frac{A_{k} \beta_{k}(t=0)}{i \tilde{\omega}-A^{\prime} / 4+A_{k} / 2},
$$

where

$$
\begin{aligned}
D(\tilde{\omega})=i \tilde{\omega}+A^{\prime} / 4 & -\frac{1}{4} \sum_{k} \frac{A_{k}^{2}}{i \tilde{\omega}-A^{\prime} / 4+A_{k} / 2}= \\
& =i \omega+\epsilon_{z}+i \omega \sum_{k} \frac{A_{k} / 2}{i \omega+A_{k} / 2}
\end{aligned}
$$

and $i \omega=i \tilde{\omega}-A^{\prime} / 4$.

1) Let us start with the case

$$
\exp (-i \hat{\mathcal{H}} t)\left|\Psi_{0}>=\alpha(t)\right| \Psi_{0}>+\sum_{k} \beta_{k}(t) \mid \Psi_{k}>
$$

which corresponds to the initial conditions $\alpha(t=0)=$ $1, \beta_{k}(t=0)=0$. Performing the Laplace transform of Eq.(45), we obtain

$$
<\Psi_{0}\left|\frac{1}{i \tilde{\omega}-\hat{\mathcal{H}}}\right| \Psi_{0}>=-i \alpha(\tilde{\omega})=1 / D(\tilde{\omega}),
$$

where we have used Eq.43) with initial conditions $\alpha(t=$ $0)=1$, and $\beta_{k}(t=0)=0$.

2) Next we consider the case

$$
\exp (-i \hat{\mathcal{H}} t)\left|\Psi_{k}>=\alpha^{k}(t)\right| \Psi_{0}>+\sum_{k^{\prime}} \beta_{k^{\prime}}^{k}(t) \mid \Psi_{k^{\prime}}>
$$

which corresponds to the initial conditions $\alpha^{k}(t=0)=0$, and $\beta_{k^{\prime}}^{k}(t=0)=\delta_{k k^{\prime}}$. Again, performing the Laplace transform of Eq.(47), we obtain

$$
\sum_{k}<\Psi_{k}\left|\frac{1}{i \tilde{\omega}-\hat{\mathcal{H}}}\right| \Psi_{k}>=-i \sum_{k} \beta_{k}^{k}(\tilde{\omega}) .
$$

It follows from Eqs. 42, 43) and the initial conditions indicated above that

$$
\beta_{k}^{k}(\tilde{\omega})=\frac{i}{4 D} \frac{A_{k}^{2}}{\left(i \tilde{\omega}-A^{\prime} / 4+A_{k} / 2\right)^{2}}+\frac{i}{i \tilde{\omega}-A^{\prime} / 4+A_{k} / 2} .
$$

Then from Eqs.48), (49) we obtain

$$
\sum_{k}<\Psi_{k}\left|\frac{1}{i \tilde{\omega}-\hat{\mathcal{H}}}\right| \Psi_{k}>=\frac{1}{4 D} \sum_{k} \frac{A_{k}^{2}}{\left(i \tilde{\omega}-A^{\prime} / 4+A_{k} / 2\right)^{2}}+\sum_{k} \frac{1}{i \tilde{\omega}-A^{\prime} / 4+A_{k} / 2}
$$

Collecting all the terms, Eqs.46), (50), we obtain

$\nu=\frac{1}{\pi} \operatorname{Im}\left[\frac{1}{D(\tilde{\omega})}\left(1+\sum_{k} \frac{\left(A_{k} / 2\right)^{2}}{\left(u+A_{k} / 2\right)^{2}}\right)+\sum_{k} \frac{1}{u+A_{k} / 2}\right]$ where $u=i \omega$. From Eq.(44) we can easily check that

$$
\frac{d}{d u} \ln D=\frac{1}{D}\left(1+\sum_{k} \frac{\left(A_{k} / 2\right)^{2}}{\left(u+A_{k} / 2\right)^{2}}\right) \text {. }
$$


Thus, from Eq.(51) we finally obtain Eq.(25) of the main text.

[1] Semiconductor Spintronics and Quantum Computing, D. D. Awschalom, D. Loss, and N. Samarth, eds. (Springer, New York, 2002).

[2] S. A. Wolf et al., Science 294, 1488 (2001).

[3] J. M. Kikkawa and D. D. Awschalom, Phys. Rev. Lett. 80, 4313 (1998).

[4] Salis et al., Phys. Rev. Lett. 86, 2677 (2001); Salis et al., Phys. Rev. B 64, 195304 (2001).

[5] D. Loss and D. P. DiVincenzo, Phys. Rev. A 57, 120 (1998).

[6] A.V. Khaetskii and Yu.V. Nazarov, Phys. Rev. B 61, 12639 (2000); 64, 125316 (2001).

[7] S.I. Erlingsson, Yu.V. Nazarov, and V.I. Fal'ko, Phys. Rev. B 64, 195306 (2001).

[8] Y.B. Lyanda-Geller, I.L. Aleiner, and B.L. Altshuler, Phys. Rev. Lett. 89, 107602 (2002).

[9] A. Khaetskii, D. Loss, and L. Glazman, Phys. Rev. Lett. 88, 186802 (2002).

[10] We note that in cases with exponential decay the spin decoherence (dephasing) time is usually denoted by $T_{2}$ $\left(T_{2}^{*}\right)$ (e.g. in the Bloch equations). E.g., in Ref. 3 it is $T_{2}^{\star}$ which is measured (due to an ensemble average over many spins). In general, $T_{2}^{\star}<T_{2}$. Since the spin decay found in the present work turns out to be non-exponential, we will hot use this notation (i.e. $T_{2}$ or $\left.T_{2}^{*}\right)$ to avoid confusion.

[11] M.I. Dyakonov and V.I. Perel, in Optical Orientation, North-Holland, Amsterdam, p. 11 (1984).

[12] M.I. Dyakonov and V.I. Perel, Zh. Eksp. Teor. Fiz., 65, 362 (1973)[Sov. Phys. JETP, 38, 177 (1973)].

[13] A. Abragam, The principles of nuclear magnetism, Oxford University Press, London, 1961.

[14] Note that we consider special nuclear configurations $\left\{I_{z}^{i}\right\}$ which we can treat analytically. We stress, however, that more general initial tensor product states which correspond to arbitrary directions of individual spins have the same time evolution as the state considered here, which was confirmed numerically in Ref.19. In contrast, a very different decay in time occurs for randomly entangled states 19. See also Sec.IV B.
[15] Since the dipolar interaction between the nuclei is neglected, the total angular momentum of the system $\vec{J}=$ $\vec{S}+\sum_{i} \vec{I}_{i}$ is conserved, where the sum runs over the entire sample (and not only over the dot). However, the corresponding angular momentum obtained with the sum running over the nuclei located within the dot is not conserved, which allows a transfer of nuclear moment to the region outside the dot via the hyperfine interaction with the electron spin.

[16] G. Burkard, D. Loss, and D. P. DiVincenzo, Phys. Rev. B 59, 2070 (1999).

[17] The nuclear spin correlator $C(t)=\left\langle I^{z}(t) I^{z}(0)\right\rangle, \vec{I}=$ $\sum_{i} \vec{I}_{i}$, can be measured directly by local NMR-type measurements such as magnetic resonance force microscopy [18]. As pointed out in Ref.19, in a subspace of given $J^{z}$ and with the electron spin pointing downwards initially, this quantity reads $C(t)=\left(J^{z}-\left\langle S^{z}(t)\right\rangle\right)\left(J^{z}+1 / 2\right)$. Then the dynamics of the total nuclear spin can be mapped out by measuring the electron spin and vice versa.

[18] For a recent overview see A. Suter, D. V. Pelekhov, M. L. Roukes, and P. C. Hammel, J. Mag. Reson. 154, 210 (2002), and references therein.

[19] J. Schliemann, A. Khaetskii, and D. Loss, condmat/0207195 (Phys. Rev. B, in press).

[20] There is an exact mathematical analogy of the expression for the DOS we have obtained here with the corresponding expression in the problem of localized phonon modes, see A.M. Kossevich, The crystal lattice: phonons, solitons, dislocations, Berlin, Wiley-VCH, 1999.

[21] See also I. A. Merkulov, Al. L. Efros, and M. Rosen, Phys. Rev. B 65, 205309 (2002). These authors have considered the problem of electron spin dephasing due to the hyperfine interaction for an ensemble of dots. They observed several time scales for this problem with different decay laws. For the initial electron spin decay which is due to the different nuclear magnetic fields realized in different dots they obtained essentially the same formula that we derived [9], Eq.38), with the same time scale $\sqrt{N} / A$. 


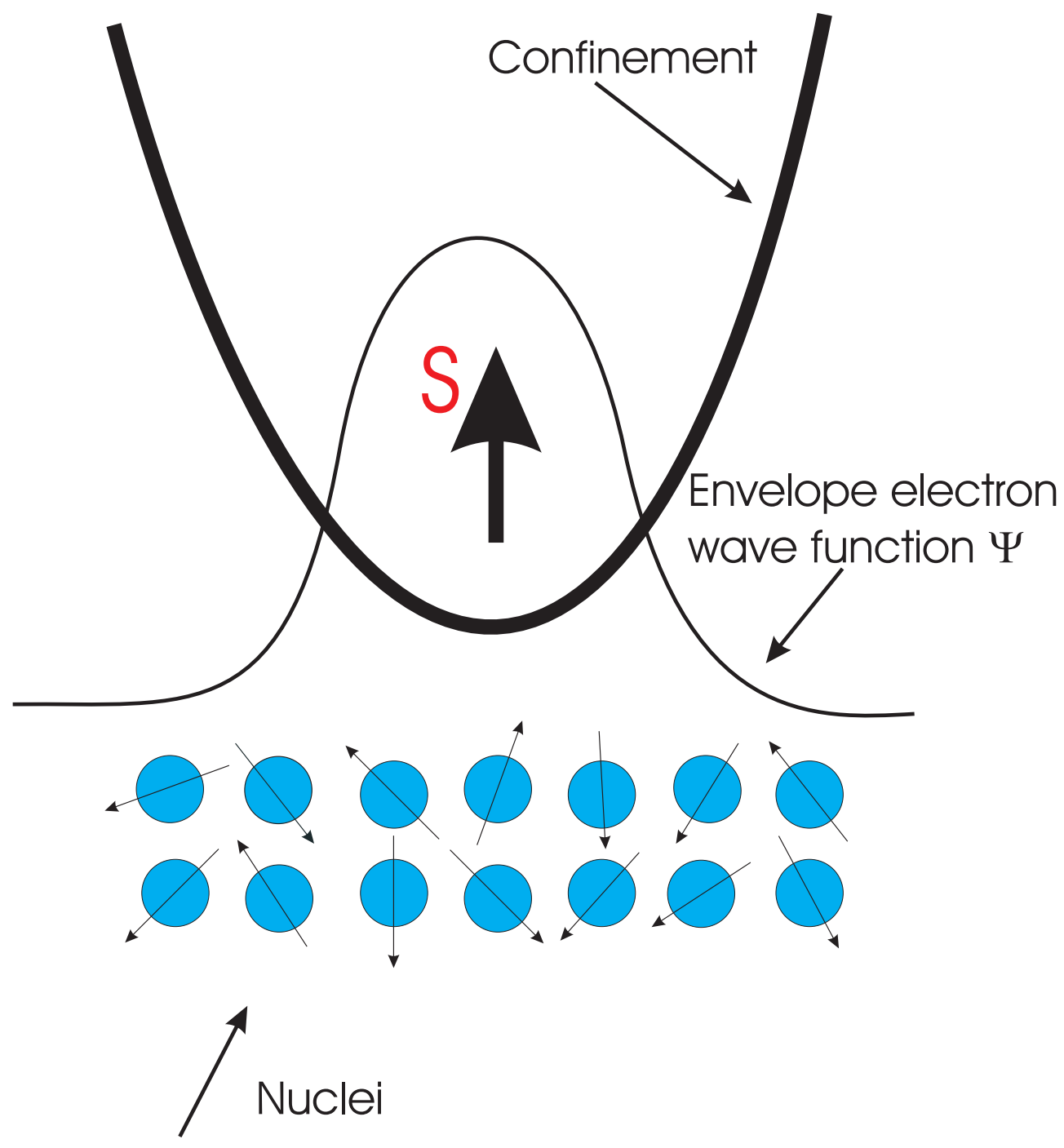

FIG. 1: A single electron spin localized in a quantum dot described by a parabolic confinement potential ( $\mathrm{x}, \mathrm{y}$ plane). The electron is assumed to be in the orbital ground state described by the envelope wave function $\Psi$, and interacts with the nuclear spins (located at $\vec{r}_{i}$ ) via hyperfine interaction $A_{i} \sim$ $\left|\Psi\left(\vec{r}_{i}\right)\right|^{2}$ which varies as function of position $\vec{r}_{i}$. 


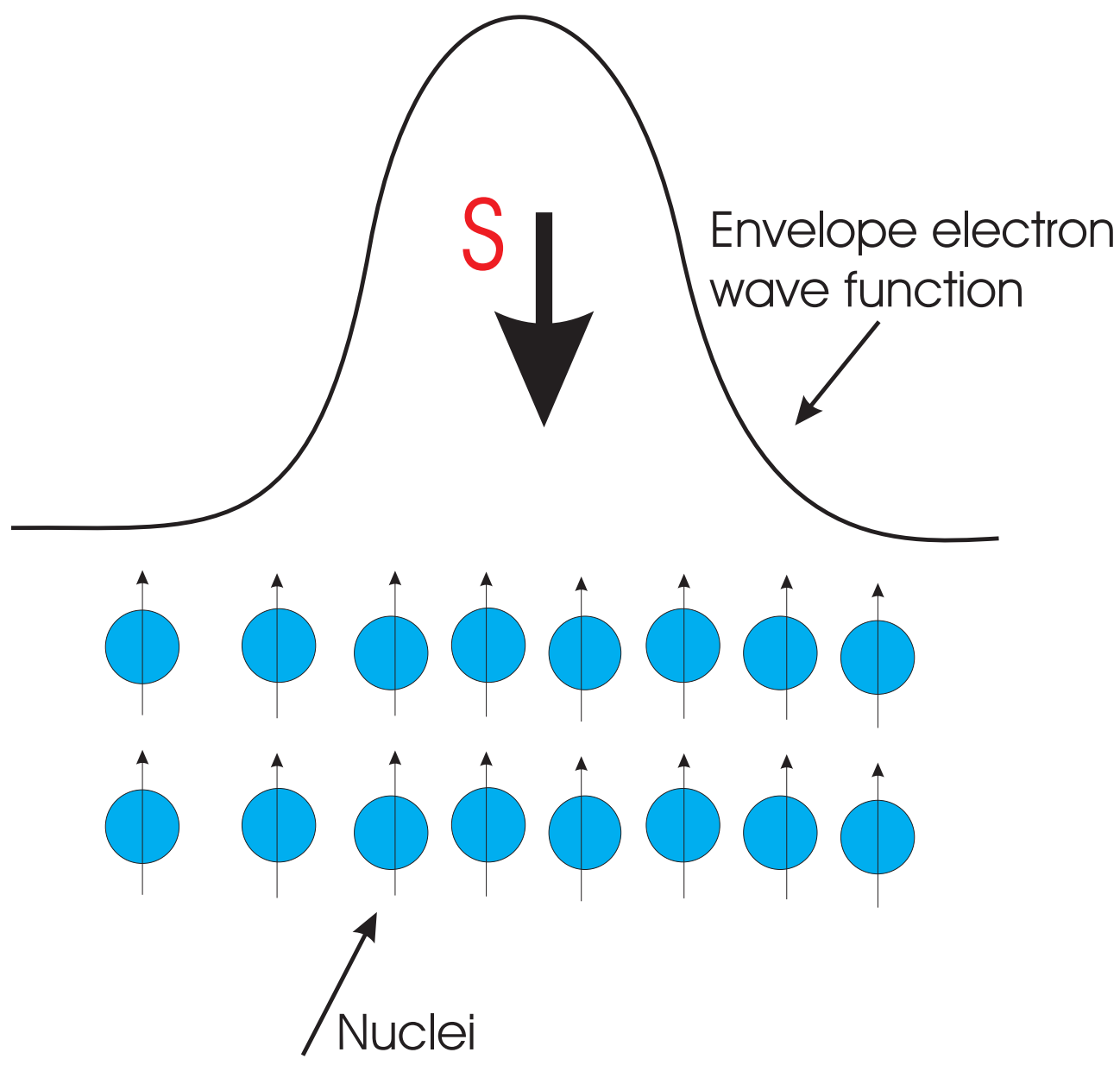

FIG. 2: The same situation as in Fig.1 but with fully polarized nuclear spins. 


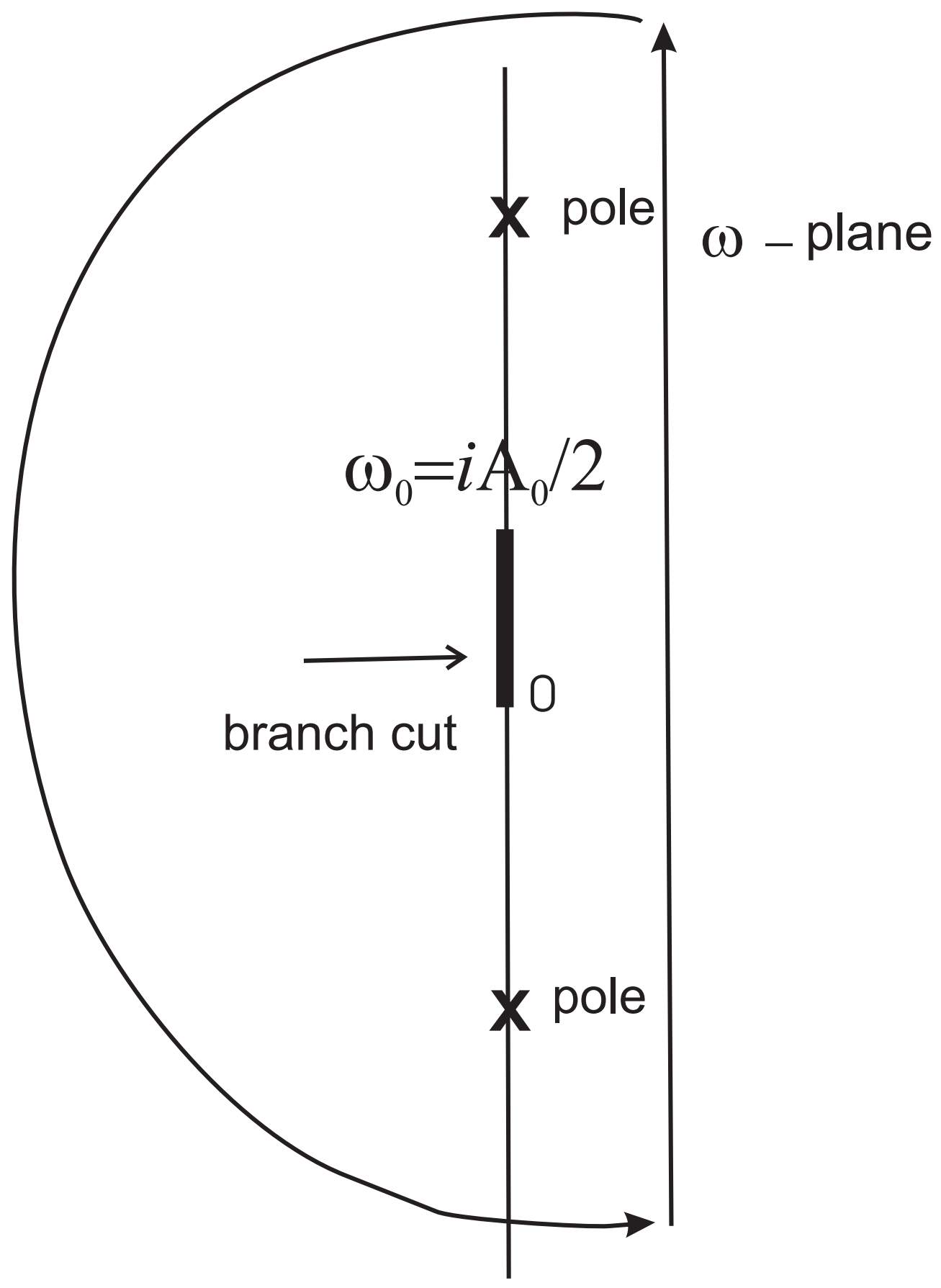

FIG. 3: The integration contour $\Gamma$ in Eq.(15), enclosing poles and branch cut. 


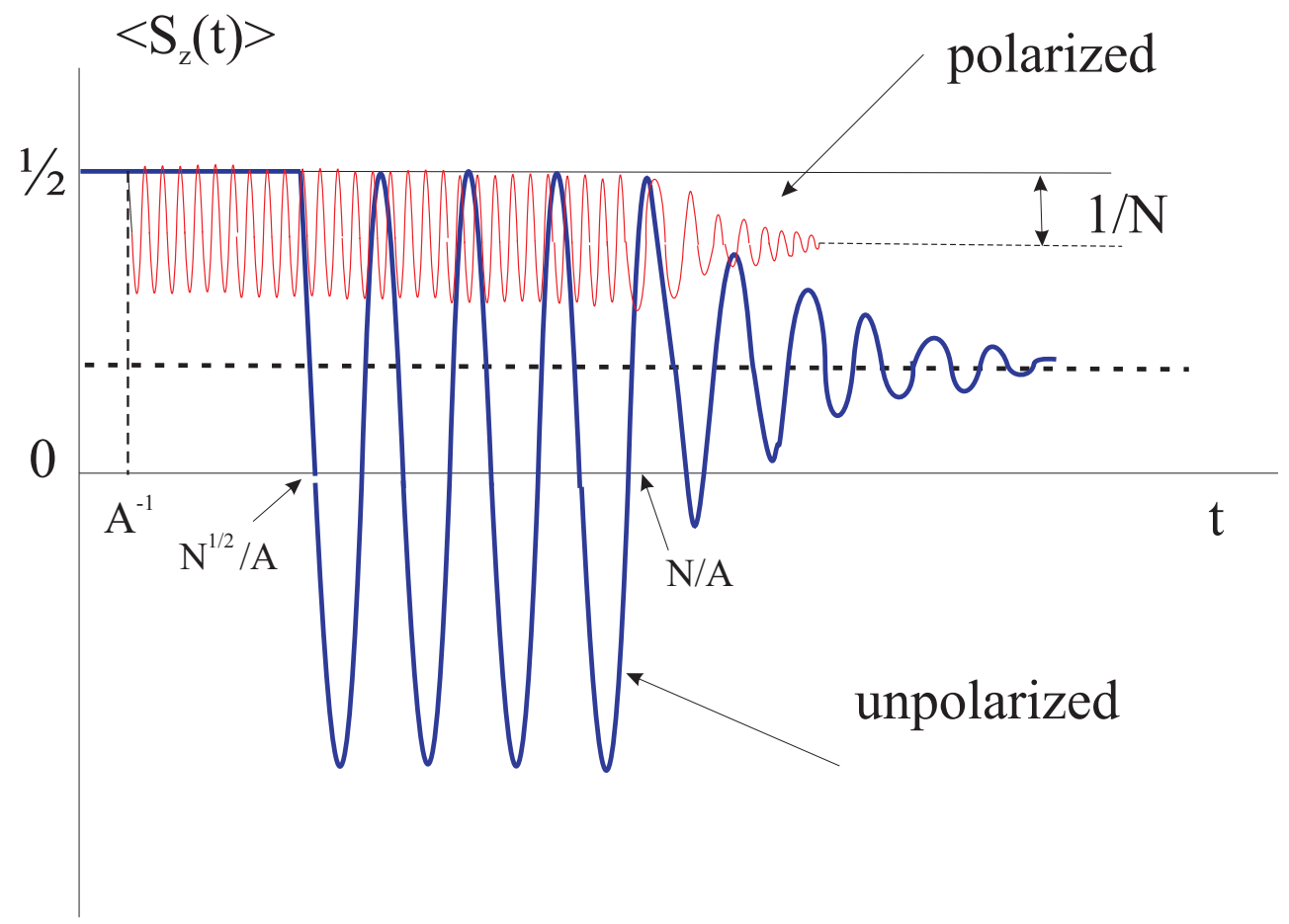

FIG. 4: Schematic dependence of $\left\langle S_{z}(t)>\right.$ on time t for the unpolarized tensor product and fully polarized nuclear states. The time scale for the onset of the decay $\sim N / A$ is the same for both cases. In the fully polarized case the magnitude of the effect is $1 / N$. The period of oscillations is of the order of $\sqrt{N} / A$ for the unpolarized and $\sim 1 / A$ for the polarized case. 


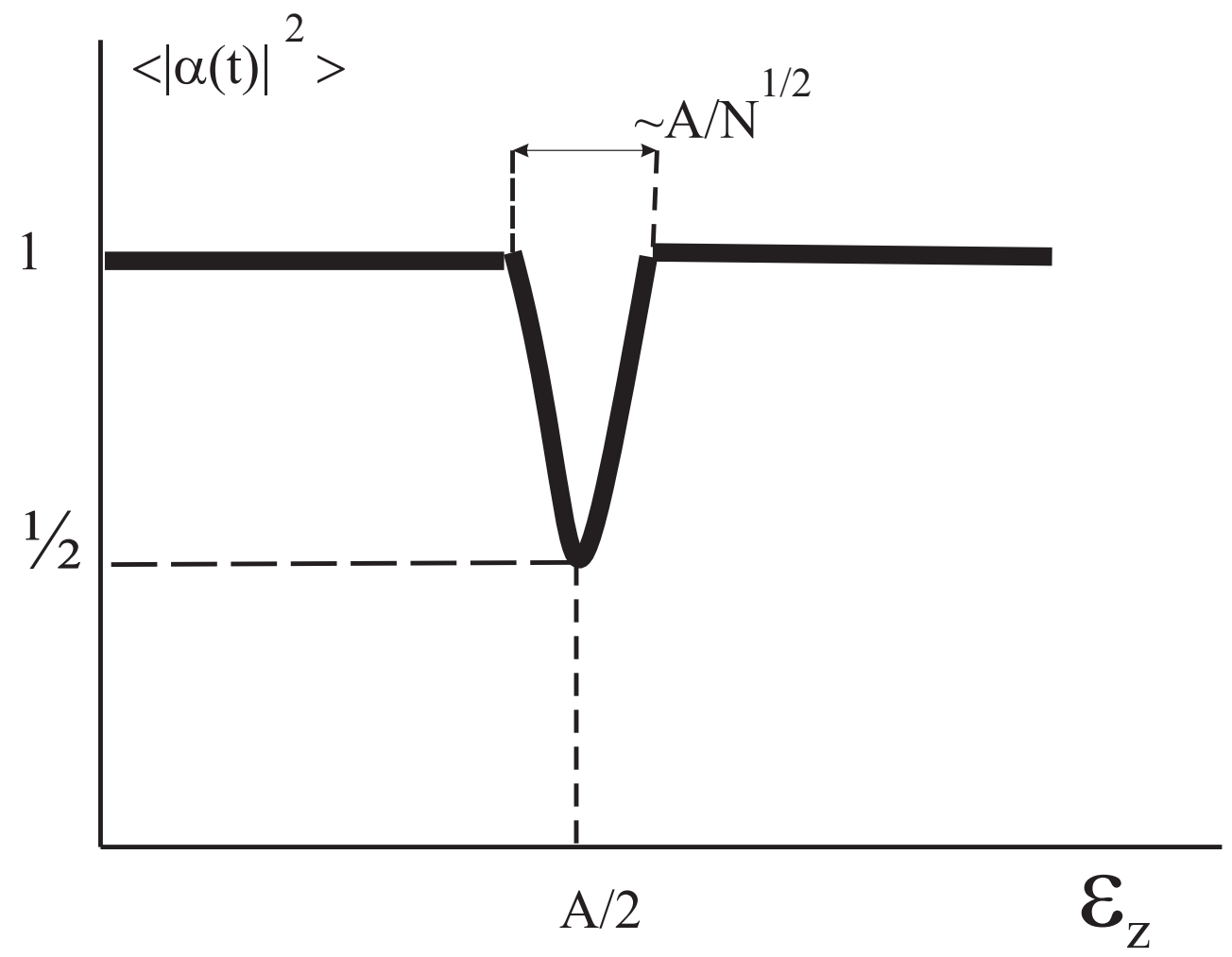

FIG. 5: The dependence of $|\alpha|^{2}$ averaged over time $(<$ $\left.|\alpha(t)|^{2}>\right)$ on the external Zeeman field $\epsilon_{z}$ for a fully polarized nuclear state. The resonance occurs at $\left|\epsilon_{z}\right|=A / 2$, and the width of the resonance is $\sim A / \sqrt{N}$, which is much smaller than the initial gap $A / 2$. 